\title{
INVESTIGADORAS MATEMÁTICAS EN COLOMBIA: DESAFÍOS, RETOS Y OPORTUNIDADES
}

\author{
Nelsy Rocío González Gutiérrez ${ }^{1}$ \\ Universidad Pedagógica y Tecnológica de Colombia-Colombia \\ nelsy.gonzalez@uptc.edu.co
}

\begin{abstract}
Resumen
Esta investigación describe el impacto académico que han tenido los aportes realizados por matemáticas colombianas en el avance del desarrollo científico del país. Se analizan los factores que han incidido durante el proceso de formación académica en los logros científicos. El estudio se realiza desde la historia social de la educación. Haciendo uso de la metodología de historia oral se analiza la influencia del entorno familiar en la elección de la carrera profesional de una matemática colombiana destacada. La estructuración se realiza desde los métodos de la historia-social, se acude a la fenomenología para efectuar la descripción-narración de los hechos. Así mismo se apoya en la hermenéutica para realizar la interpretación de la información recolectada de entrevistas. El estudio históricocomparado del desarrollo de los estudios en matemáticas realizados por nuestra participante nos permite develar la situación relativa de géneros, tanto académica como social, que tuvo lugar y nos permite concluir que el avance académico obtenido por la investigadora objeto de estudio estuvo motivado por iniciativa propia, así como por vivencias en su formación escolar inicial. Así mismo, el avance académico obtenido en el doctorado en matemáticas constituye un logro que le ayuda a cumplir el desafío de ser un ejemplo de vida que inspirará a futuras matemáticas.
\end{abstract}

Palabras Clave: Historias de vida, investigadora, mujeres, matemáticas.

1. Docente Asociada a la Escuela de Matemáticas y Estadística de la UPTC y estudiante del Doctorado en Ciencias de la Educación de la Red de Universidades RUDECOLOMBIA, CADE-UPTC. 


\title{
MATHEMATICAL RESEARCHERS IN COLOMBIA: CHALLENGES AND OPPORTUNITIES
}

\begin{abstract}
This investigation describes the academic impact of the contributions made by Colombian mathematics on the advance of the country's scientific development, analyzing the factors that have impacted scientific achievements during the academic formation process. The study is made from the perspective of the social history of education. Using the methodology of oral history, an analysis is made of the influence of the family environment on the professional career choices of a prominent Colombian mathematician. Structuring is done with social-historical methods, and phenomenology is used to create a description-narration of the facts. The work relies on hermeneutics to perform the interpretation of the information gathered from interviews. The historical-comparative study of the development of mathematics carried out by our participant allows us to demonstrate the relative gender situation (both academically and socially speaking) that occurred and brings us to the conclusion that the academic advance obtained by the mathematician was motivated by their own initiative, as well as early education experiences. Likewise, the academic advance obtained in the course of their doctorate in mathematics is an achievement that helps her meet the challenge of being an example that will inspire future mathematicians.
\end{abstract}

Keywords: Life stories, investigator, women, math.

\section{PESQUISADORAS MATEMÁTICAS NA COLOMBIA: DESAFITOS, RETOS E OPORTUNIDADES}

\begin{abstract}
Resumo
Esta pesquisa descreve o impacto acadêmico que as contribuições feitas pela matemática colombiana tiveram no avanço do desenvolvimento científico do país. Os fatores que impactaram durante o processo de formação acadêmica sobre conquistas científicas são analisados. 0 estudo é feito a partir da história social da educação. Utilizando a metodologia da história oral, analisa-se a influência do ambiente familiar na escolha da carreira profissional de uma proeminente matemática colombiana. A estruturação é feita a partir dos métodos histórico-sociais, a fenomenologia é utilizada para fazer a descrição-narração dos fatos. Da mesma forma, confia na hermenêutica para realizar a interpretação das informações coletadas nas entrevistas. 0 estudo histórico-comparativo do desenvolvimento de estudos em matemática feita por nossa participante nos permite compreender a situação relativa de gêneros, tanto academicamente e socialmente, que teve lugar e permite-nos concluir que o progresso acadêmico obtido pela investigadora objeto de estudo foi motivado por iniciativa própria, bem como experiências em sua formação escolar inicial. Da mesma forma, o avanço acadêmico obtido no doutorado em matemática é uma conquista que o ajuda a enfrentar o desafio de ser um exemplo de vida que inspirará futuras matemáticas.
\end{abstract}

Palavras-chave: Histórias de vida, pesquisador, mulheres, matemática. 


\section{Introducción}

La investigación se centró en analizar la historia de vida de una investigadora en ciencias matemáticas en la universidad colombiana, dentro de un paradigma de ciencia pura que privilegia el carácter masculino sobre lo femenino ${ }^{2}$ y uno educacional que superpone al matemático sobre el licenciado en matemáticas.

El periodo de estudio de esta investigación, que se circunscribe al desarrollado en la Tesis Doctoral de la autora ${ }^{3}$, comprende gran parte de la segunda mitad del siglo XX, inicia en el año 1961, por ser el año en que se gradúan los primeros matemáticos en Colombia y finaliza en el año 2000 cuando se gradúan las tres primeras mujeres doctoras en matemáticas formadas en la universidad colombiana, sus títulos de maestría y doctorado les fueron otorgados en la Universidad Nacional de Colombia en Bogotá, institución pionera en estudios de matemáticas en el país ${ }^{4}$. Una de estas mujeres es la protagonista de la historia de vida que analizamos en este trabajo.

Conscientes de la relevancia que tiene la influencia de la sociedad en el desarrollo profesional del ser humano, consideramos que es de vital importancia conocer el entorno social y cultural existente durante el período de tiempo que comprende esta investigación. Conocer el hábitat que rodeó a las primeras investigadoras colombianas de matemáticas ayudará a comprender el por qué los acontecimientos, ocurrieron cómo lo hicieron. Se precisa que solo a mediados del siglo XX la larga disputa de las mujeres para ejercer sus derechos de ciudadanía empieza a dar frutos ${ }^{5}$. Es conocido que, para la toma de decisiones, las mujeres vivían bajo el dominio total, primero de su padre o hermanos varones y luego, al casarse pasaban a depender totalmente de su marido ${ }^{6}$.

Por otro lado, pareciera "natural" que al realizar una revisión general en la historia de la ciencia no se vislumbre tácitamente en ella la participación femenina7. Esto no se debe a que las mujeres se hayan mantenido aisladas de los procesos de generación de conocimiento científico ${ }^{8}$, sino más bien al hecho de una historia de la ciencia contada desde la óptica de los hombres.

2. Sandra Harding, The Science Question in Feminism (Ithaca, United States: Cornell University Press, 1986), 296.

3. Nelsy Rocío González Gutiérrez, "Investigadoras en Ciencias Matemáticas en la universidad colombiana. 1961-2000", Tesis de Doctorado en Ciencias de la Educación. RUDECOLOMBIA-UPTC, 2017.

4. Entrevista a Mantilla Prada, Eduardo, Bogotá D.C., 22 de enero de 2016.

5. "Los intensos años sesenta, capítulo del libro de Álvaro Tirado Mejía", El Tiempo, https://www. eltiempo.com/archivo/documento/CMS-14481235 (12 de mayo de 2016).

6. Diana Elvira Soto Arango, La escuela rural en Colombia: historias de vida de maestras. Mediados del siglo XX (Tunja: grupos HISULA, ILAC, SHELA, 2014), 51-58.

7. Margaret Alic, El Legado de Hipatia. Historia de las mujeres en la ciencia desde la antigüedad hasta fines del siglo XIX (México DF., Siglo XXI, 2005), 246.

8. Gonzalo Gallo G. 'Mujeres'. Periódico El Tiempo (2000b), Redactado el 20 de julio de 2000, versión Online, http://www.eltiempo.com/ archivo/documento/MAM-1270739 (19 de mayo de 2016). 
Finalmente, la voz de nuestra investigadora nos confirma que el fenómeno de invisibilidad de las mujeres matemáticas se encuentra aún latente en nuestra so$\operatorname{ciedad}^{9}$. Este suceso también se sigue manteniendo en el contexto internacional ${ }^{\mathbf{1 0}}$, basta con revisar los artículos de prensa actuales para darse cuenta que todo el arduo trabajo y aportaciones científicas que han realizado las mujeres matemáticas $^{\mathbf{1 1}}$ no han sido suficientes para sacar de la penumbra la capacidad intelectual del género femenino.

Prueba de ello, se muestra en el artículo conmemorativo con ocasión del día de la mujer publicado en el Diario El País ${ }^{12}$. En éste se hace alusión al reconocimiento que se le realizó a la matemática de la NASA, Katherine Johnson, a sus 98 años, en el Dolby Theatre, durante la celebración de la 89a edición de la entrega de los premios Oscar, de cine, junto al reparto de la película "Figuras Ocultas" de Theodore Melfi (2016), el 26 de febrero de 2017. La película en mención es un tributo realizado con el propósito de que el mundo conociera el trabajo silencioso de la matemática Katherine Jhonson, junto a otras mujeres afroamericanas en la NASA. Katherine Johnson fue una de las primeras mujeres en trabajar en la agencia espacial estadounidense y una de las primeras mujeres negras en ocupar un puesto de importancia. Ayudó a describir las órbitas de los cohetes del primer programa espacial estadounidense tripulado (Mercury) y del tan famoso Apolo 11 que llevó al ser humano a la Luna. Todo este trabajo fue desarrollado en una época en la que además de la discriminación de género, todavía había segregación racial ${ }^{13}$.

Resaltamos que a nivel internacional la problemática de dar visibilidad a la investigación científica de mujeres matemáticas ha empezado a tener eco en la comunidad, por este motivo, se han creado agremiaciones que estimulan dicha labor científica, una de ellas es la Association for Women in Mathematics (AWM) ${ }^{\mathbf{1 4}}$.

9. Diario El País. 'Por primera vez en la historia, una mujer gana la medalla Fields de Matemáticas. La iraní Maryam Mirzakhani, de 37 años, recibe el que se considera el Nobel de estas ciencias. Entre los otros tres galardonados, un brasileño: primer latinoamericano en obtener la distinción'. https:// elpais.com/sociedad/2014/08/13/actualidad/1407915530_921490.html. Redactado el 13 de agosto de 2014. THE SEOUL ICM 2014 (AFP).

10. UNESCO (1998), 'Programa La Mujer y la Ciencia', Organización de las Naciones Unidas para la Educación la Ciencia y la Cultura. Disponible en http://www.unesco.org/new/es/natural-sciences/ priority-areas/gender-and-science/for-women-in-science-programme/ (05 de agosto de 2014).

11. Conversatorio: 'Mujeres matemáticas en Colombia. Algunos testimonios'. Dentro del XXI Congreso Colombiano de Matemáticas realizado en la sede Bogotá de la Universidad Nacional de Colombia entre el 5 y el 9 de junio de 2017. Disponible en https://scm.org.co/scm2017/2017/07/18/ conversatoriomujeres-matematicas-en-colombia-algunos-testimonioso-lineal-y-ecuaciones-diferencialesparciales-2017-2/

12. Edith Padrón y Ágata A. Timón. ‘¿Dónde están las mujeres matemáticas?’ Día Internacional de la Mujer. Sección Café y Teoremas, El País, Edición América, del jueves 9 de marzo de 2017. https://elpais.com/ elpais/ciencia/1488907524_284402.html (07 de marzo de 2017).

13. Roger Cooke, The History of Mathematics, A Brief Course (New Jersey: John Wiley \& Sons, Inc., 2005).

14. La información completa de esta asociación se encuentra en el sitio web: https://sites.google.com/ site/awmmath/home 
La Asociación de Mujeres en Matemáticas es una organización estadounidense sin ánimo de lucro, fundada en 1971, con el propósito de animar a las mujeres y las niñas a estudiar y a tener carreras activas en las ciencias matemáticas, así como promover la igualdad de oportunidades y la equidad en el trato de las mujeres y las niñas en estas ciencias.

De igual forma, la Organización de Naciones Unidas, recientemente estableció el día 11 de febrero como Día Internacional de la Mujer y la Niña en la Ciencia15. Entre los propósitos de creación de este Día Internacional se tienen: instar a que se asuma el compromiso de poner fin a los prejuicios; exhortar en la necesidad de incrementar la inversión en educación en ciencia, tecnología y matemáticas para todas las mujeres y las niñas; y a insistir en la necesidad de que a las mujeres y niñas se les ofrezcan oportunidades para mejorar sus perspectivas de carrera y su desarrollo profesional ${ }^{\mathbf{1 6}}$ a más largo plazo, para que todas las personas puedan beneficiarse de sus innovadoras contribuciones en el futuro.

Por lo anterior, consideramos muy importante conocer acerca de las cualidades y experiencias de las mujeres ${ }^{\mathbf{1 7}}$, como la que referenciamos en esta investigación, debido a que ellas representan al espíritu de la matemática como ciencia y condensan nuestras esperanzas para un futuro en el cual hombres y mujeres se desempeñen en total equidad.

Esta investigación es de corte cualitativo ${ }^{\mathbf{1 8}}$ en cuanto los datos a analizar son de carácter social y serán analizados de manera comprensiva e interpretativa para explorar las relaciones sociales y describir la realidad tal como la experimentan sus protagonistas. El método ${ }^{19}$ se centró en la historia social de la educación con la metodología de historia de vida, y la entrevista ${ }^{20}$ como instrumento. En síntesis, para realizar la interpretación (hermenéutica ${ }^{21}$ ) de la situación relativa a los géneros en los estudios de matemáticas en Colombia, en esta investigación se parte del esquema previo determinado por la Historia social en la educación, lo cual permite, mediante la historia oral, describir los hechos, tal y como son, en forma narrativa (fenomenología).

15. Organización de Naciones Unidas. Día Internacional de la Mujer y la Niña en la Ciencia, 11 de febrero. Disponible en http://www.un.org/es/events/women-and-girls-in-science-day/.

16. Renée Clair, La formación científica de las mujeres. ¿Por qué hay tan pocas científicas? (Barcelona: Ediciones Unesco, 1994).

17. SCM (2015). Foro: Las Matemáticas en Colombia, Universidad Nacional de Colombia sede Manizales y Sociedad Colombiana de Matemáticas. SCM, XX Congreso Colombiano de Matemáticas, julio 21-24. Disponible en https://www.youtube.com/watch?v=QQh3HG9tNs4\&amp;feature=youtu.be (27 de agosto de 2017).

18. Vasilachis et al., Estrategias de investigación cualitativa (Barcelona: Gedisa, S.A., 2006).

19. John W. Creswell, Qualitative inquiry research desing. Choosing among five approaches, first ed (United States of America: SAGE Publications, Inc., 2013).

20. Michael Appel, 'La entrevista autobiográfica narrativa: Fundamentos teóricos y la praxis del análisis mostrada a partir del estudio de caso sobre el cambio cultural de los Otomíes en México [127 párrafos]', Forum Qualitative Sozialforschung / Forum: Qualitative Social Research 6(2). 2005. 1-35. Disponible en http://nbn-resolving.de/urn:nbn:de:0114-fqs0502160

21. Aquí los fenómenos son vistos como textos que adquieren una existencia independiente del autor. 


\section{LAS VOCES ${ }^{22}$ DE LAS INVESTIGADORAS MATEMÁTICAS COLOMBIANAS}

Según la Inter-American Network of Academies of Sciences - IANAS (2015) ${ }^{23}$, en la actualidad, las mujeres siguen contando con escasa representatividad en el campo de la ciencia, y la sociedad no está reconociendo, por completo, el potencial del talento humano y la creatividad de las mujeres y hombres de ciencia ${ }^{24}$.

En consecuencia, en esta investigación se realizaron una serie de entrevistas semi-estructuradas a científicas colombianas destacadas ${ }^{25}$, con la esperanza de que sus voces, vivencias, experiencias, así como su trayectoria profesional inciten a más mujeres a considerar la elección de su carrera en la ciencia, y en especial en la Matemática.

Mirar las vidas de estas mujeres es una manera de sacar a la luz aquello que se ha ocultado durante mucho tiempo. Ayuda a revelar cómo incluso la más "objetiva" de las disciplinas no puede estar inmune a los prejuicios sociales. También, nos ayuda a entender cómo el género ${ }^{26}$ influye en quién se convierte en matemático(a), cómo influye en la experiencia de los(as) matemáticos(as) e indirectamente cómo influye en la disciplina misma.

Además, sólo al hacer visibles las barreras invisibles, se puede esperar la creación de un cambio real y duradero ${ }^{27}$. Quizá, hay quienes consideren esto como un imposible, que las dificultades son agotadoras y que se tendrá que sacrificar mucho para tener éxito como matemática, pero al conocer las historias de mujeres activas y brillantes, quienes con compromiso, perseverancia, años de preparación, confianza en sus capacidades, tenacidad para alcanzar el éxito y capacidad de adaptación a nuevos entornos, se vislumbra que ellas han trazado un camino que más mujeres ${ }^{28}$ pueden seguir para poder lograr la recompensa final: una vida plena, gozando del respeto y reconocimiento de sus colegas y comunidad académica en general.

22. Ranahit Guha, Las voces de la historia y otros estudios subalternos (Barcelona: Editorial Crítica, 2002).

23. Inter-American Network of Academies of Sciences - IANAS (2015), 'Jóvenes científicas: Un futuro brillante para las Américas', http://www.ianas.org/women_2015/young_women_scientists/ librojovenes-cientificas.pdf. Traducido por Suzanne D. Stephens, Impreso en México ISBN: 978-607-837924-8, versión Online (16 de mayo de 2016).

24. Viky Frías Ruiz, ed., Las mujeres ante la ciencia del siglo XXI (Madrid: Instituto de Investigaciones Feministas, Editorial Complutense S.A., 2001), 163-228.

25. En total se entrevistó a tres matemáticas colombianas reconocidas por realizar aportes en investigación.

26. Joan W. Scott (1986), 'Gender: A useful category of historical analysis', The American Historical Review 91(5), 1053 -1075. https://racismandnationalconsciousnessresources

27. Boaventura De Sousa Santos y Maria Paula Meneses. Epistemologías del Sur, Cuestiones de antagonismo (Madrid: Ediciones Akal, 2014), 544.

28. Secretaría de Naciones Unidas (1995), 'Informe de la cuarta conferencia mundial sobre la mujer', http://www.un.org/womenwatch/daw/beijing/pdf/Beijing\% 20full\%20report\%20S.pdf (15 de enero de 2011). 
Para guiar la entrevista ${ }^{29}$ se diseñó un cuestionario que contenía algunas preguntas orientadoras básicas. Así mismo, durante el desarrollo de la entrevista se procuró dejar libertad para formular preguntas independientes generadas por la interacción personal, con el propósito de sacar a la luz aspectos que destaquen la individualidad de cada una de nuestras protagonistas.

Del análisis realizado a las entrevistas efectuadas se estructuraron las historias de vida de cada una de las investigadoras, una de las cuales será el objeto central de este artículo y que a continuación se presenta.

\section{SONIA MARLENI SABOGAL PEDRAZA. UNA VIDA DIGNA DE ADMIRAR}

Los temas de interés investigativo de la doctora Sonia Sabogal están relacionados con Geometría fractal, propiedades de auto-semejanza en Topología y algunas extensiones de la dualidad de Stone. Su línea de investigación en el área de Topología la llevó a convertirse en un referente de esta área en el país y en el exterior.

Nació el primero de diciembre de 1962, en Bogotá D.C., Colombia. Madre de dos hijos, Esteban y Laura, y abuela de dos nietas, Mariana y Safira. Sonia cuenta que la influencia de su profesor de Trigonometría y Física del colegio resultó vital a la hora de elegir su futuro profesional, fue él quien notó que su habilidad con los números y su rigor analítico era evidente. "El que me animó fue un profesor, el que me dio Física y Trigonometría y luego Física y Cálculo. Él fue el que prácticamente me obligó a presentarme, porque yo no tenía eso en mente"30. Inicialmente Sonia no encontró su motivación para estudiar matemáticas en su familia, pues creció en un ambiente familiar que careció de oportunidades para realizar

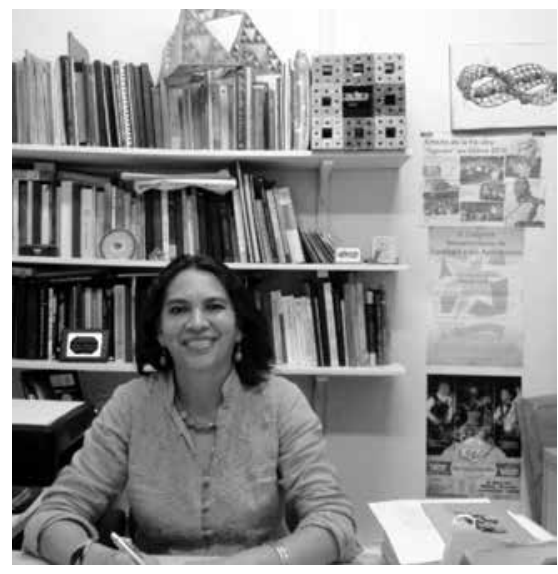

Figura 1. Sonia Marleni Sabogal Pedraza

Fuente: Archivo personal. una carrera profesional. Su padre, de origen campesino, no culminó el bachillerato, estudió un curso técnico en el SENA (Servicio Nacional de Aprendizaje ${ }^{31}$ ), el cual le permitió desempeñarse laboralmente como empleado en una fábrica. Su madre, no tuvo posibilidades de desarrollo profesional y dedicó todos sus esfuerzos a la

29. Jean-Pierre Deslauriers, Investigación Cualitativa. Guía Práctica, Universidad Tecnológica de Pereira. RUDECOLOMBIA, Traducción de Miguel Ángel Gómez, 2004.

30. Respuestas proporcionadas por la protagonista en entrevista realizada en la ciudad de Bucaramanga el día 8 de mayo de 2016.

31. EL SENA es un establecimiento público del orden Nacional adscrito al Ministerio del Trabajo de Colombia. Ofrece formación gratuita a colombianos que se benefician con programas técnicos, tecnológicos y complementarios enfocados en el desarrollo económico, tecnológico y social del país. 
crianza de sus siete hijos, así como a las labores propias del hogar. Su padre tenía el pensamiento patriarcal de la época que le tocó vivir, heredado de personajes influyentes de siglos atrás ${ }^{32}$. Por tal razón, no veía en el estudio, la forma de crecimiento personal y profesional de sus hijos y con menores expectativas en sus cinco hijas. Aunque los siete hermanos terminaron el ciclo completo de bachillerato, únicamente Sonia prosiguió y culminó estudios universitarios. "Yo no pensaba realmente estudiar en la universidad porque la expectativa en mi familia no era esa, menos pues, porque era mujer. Según mi papá las mujeres llegaríamos a cuarto de bachillerato, que se llamaba en esa época, y no más... y, luego estudiar una secretaría o casarse, o algo así. Mi mamá en cambio, y eso era a veces motivo de pequeños enfrentamientos entre ellos, siempre hacía énfasis en la importancia de leer y estudiar; recuerdo que con frecuencia decía que la lectura y el estudio eran el mejor tesoro que una persona podía poseer, así ella, mi mamá, también fue una importante influencia que me animó a seguir estudiando"33.

Cursó sus estudios de básica primaria en la Concentración Distrital Santa Ana Sur, una escuela pública cerca de su lugar de residencia en Bogotá, su ciudad natal. El bachillerato lo realizó en el Colegio Isabelita Tejada, un colegio privado femenino y según Sonia pequeño, "era privado, pero era como un colegio de barrio". El ambiente escolar era tranquilo y entre sus compañeras generalmente no se vivían ningún tipo de tensiones derivadas por rivalidades inherentes al buen rendimiento académico. Sonia fue una estudiante sobresaliente, no era la mejor pero siempre estaba entre las tres mejores del curso.

Aun cuando en un principio Sonia no encontró su principal aliento para instruirse profesionalmente en el seno familiar, empezó sus estudios de formación universitaria en 1980, en ese año ingresó al programa de Licenciatura con estudios principales en Matemáticas en la Universidad Pedagógica Nacional ${ }^{34}$, en Bogotá, estudios que terminaría en el año 1984. Por aquella época los programas de formación de licenciados tenían una duración de cuatro años. Durante sus estudios de licenciatura se destacó por su excelente rendimiento académico, siendo merecedora del estímulo académico de Matrícula de Honor ${ }^{35}$, distinción que otorgaba la Universidad semestralmente a los estudiantes que se distinguían por su alto rendimiento académico.

De aquella época, Sonia recuerda que el proceso de transición del colegio a la universidad para ella fue algo difícil, sin embargo, dada su tenacidad y sus

32. María Isabel Lafuente Guantes, "El proyecto educativo-ilustrado de Kant", Revista Historia de la Educación Latinoamericana, No. 13 (2009): 241-264. http://www.redalyc.org/articulo. oa?id=86912384013 (16 de mayo de 2016).

33. Entrevista a Sabogal Pedraza, Sonia Marleni, Bucaramanga, 8 de mayo de 2016.

34. Juan Carlos Garzón Barreto (s.f), "El proceso de consolidación de la Universidad Pedagógica Nacional Femenina en el marco de la contrarreforma educativa de los años 50", Publicaciones de la Universidad Pedagógica Nacional. http://www.pedagogica.edu.co/storage/rce/articulos/rce36-37_05ensa.pdf (16 de mayo de 2016).

35. La Matrícula de Honor consistía en la exoneración del pago de matrícula a aquellos estudiantes que obtuviesen un promedio superior a 4.5 (cuatro punto cinco). 
fuertes condiciones de adaptabilidad salió avante en dicho proceso "Los primeros semestres ... ¡ difícil!', para mí el cambio de colegio a la universidad fue difícil por diferentes razones. Yo estaba chiquita de todas maneras, tenía dieciséis años y yo siempre había estudiado en el colegio del barrio, yo estudiaba en el sur y luego pasé a estudiar a la Pedagógica, y la Pedagógica era en la Avenida Chile en la 72, entonces coger bus, atravesar el centro... yo llegaba como asustada a las clases. Pero luego ya, cuando cogí confianza y vi que me estaba yendo bien, entonces ya era muy chévere por estar con los compañeros y todo lo que uno hace con los compañeros, no solo estudiar, sino que jugábamos y fiestas y todo eso..." En la cohorte de Sonia iniciaron sus estudios de licenciatura cuarenta estudiantes, en proporción eran más mujeres que hombres, no obstante, al finalizar se invirtió dicha proporción: se graduaron más hombres que mujeres. De la mayoría de sus profesores del pregrado guarda gratos recuerdos, les reconoce su pasión por la matemática; su honestidad y sencillez, en particular guarda un afecto especial a su profesor de Geometría euclidiana: Emiliano Palacios. Durante esa etapa de formación únicamente tuvo una profesora, los demás docentes fueron todos hombres "en el pregrado tuve una profesora, doña Cecilia Leguizamón, ella me daba seminario de docencia o algo así, ella era muy buena, la recuerdo mucho, pero fue la única mujer en el pregrado". Así como tuvo docentes que para ella fueron ejemplo a seguir, también existieron otros docentes que le provocaron sentimientos totalmente opuestos, aquí evoca a un docente que en palabras de Sonia: "era deshonesto porque le encontramos errores en la clase y él no los aceptaba o se salía por la tangente, incluso nosotros nos quejamos...". Aun cuando Sonia jamás sintió algún tipo de discriminación por su condición de ser mujer en un ambiente académico donde predominaba la supremacía masculina, sí conoció casos en los que tales tipos de discriminación fueron notorios.

Una vez titulada como docente de matemáticas y estando en ejercicio de su profesión en un colegio, motivada por su curiosidad y sus ganas de mantenerse activa académicamente junto con una amiga que había sido compañera de estudio de la licenciatura, deciden continuar con su proceso de formación posgraduada. "... con mi amiga nosotras trabajábamos en colegio y con ella dijimos... hagamos una maestría, y nos fuimos a la Nacional a averiguar y nos encontramos con Myriam Campos, ella llamó a Januario Varela y nos dijo sí, sí, hagan esto, hagan lo otro... y fue todo más por curiosidad, por hacer algo más...".

En enero de 1984 es admitida al programa de Maestría en Ciencias Matemáticas de la Universidad Nacional de Colombia sede Bogotá, obteniendo su título de Magister en Ciencias Matemáticas en el año 198836. El proceso formativo en la maestría fue un gran reto para Sonia, en primer lugar, porque era egresada de un programa de formación de docentes que en gran parte difería del programa de

36. Secretaría Facultad de Ciencias - Universidad Nacional de Colombia (2016), 'Base de datos de graduados(as) de la carrera de Matemáticas y de los posgrados de Maestría y Doctorado en Matemáticas', Secretaría Facultad de Ciencias. Contiene los datos de egresados desde 1961 hasta 2016. 
formación de matemáticos. Sin embargo, para Sonia el conocimiento adquirido en su pregrado le dio bases para poder llevar a cabo sus estudios de maestría en matemática, venciendo los prejuicios existentes en el "pobre rigor matemático" de los programas de licenciatura en matemáticas frente a los de una carrera de matemáticas: "Ios licenciados estábamos en desventaja con los matemáticos puros, y si recuerdo, incluso profesores de la Nacional, ese era otro prejuicio digamos un poco bien fundado porque uno como licenciado veía más componentes de la parte didáctica, por ejemplo nosotros no veíamos sino un curso de topología, no veíamos análisis, bueno unas cosas que veían los matemáticos, entonces un profesor nos decía que los licenciados entraban con el estigma de licenciado, o sea que estábamos en desventaja. Al principio si nos tocaba duro... entramos de la licenciatura como tres, nos tocó estudiar más, pero se pudo".

Otra limitante que tuvo que soslayar fue el poder conjugar el cambio de prioridades que le trajo consigo la maternidad ${ }^{37}$. Fue madre de su primer hijo apenas iniciaba el proceso de sus estudios de maestría. "En el primer semestre de la maestría yo quedé embarazada de mi hijo, entonces yo dije ;no... hasta aquí llegué, no... ya no puedo más! Pero justamente la directora del programa de maestría en esa época, que era Myriam Campos, se enteró de unas becas de un acuerdo que existía en esa época entre el BID (Banco Internacional de Desarrollo), creo... Colciencias o Icetex y la Universidad Nacional, ella me mandó llamar y me dijo que aplicara a una de esas becas y pues apliqué y la gané y eso fue lo que me permitió seguir. Era relativamente buena la beca. Me permitió estudiar y ver a mi hijo y sin trabajar porque era una de las exigencias de la beca. Todo el posgrado lo hice con la beca".

En este ciclo de formación profesional Sonia también encontró docentes que fueron claves en la culminación exitosa de sus objetivos académicos, entre estos docentes, ella recuerda con especial aprecio a los profesores Januario Varela, Yu Takeuchi, Carlos Ruiz y Víctor Albis, este último actuó como director de su tesis de maestría. En todos ellos Sonia admiraba su espontaneidad, así como la vehemencia con la que se dedicaban a interactuar en ese fascinante mundo de las matemáticas. Al preguntarle a Sonia por sus profesoras durante este período, ella comenta que sólo tuvo una docente mujer, la profesora Myriam Campos, quien le orientara la asignatura Álgebra Lineal y a quién además le guarda especial estima: "en el posgrado pues Myriam Campos, quien fue muy solidaria conmigo". Igualmente, Sonia también expresa gran admiración por otra docente: "yo admiraba mucho, aunque ella no fue profesora mía, pero la conocí y compartí con ella en otros ambientes, a Victoria..." (se refiere a María Victoria Gutiérrez Santos, muy reconocida académicamente entre sus colegas, pero quien lastimosamente falleciera en un trágico accidente a principios de la década de los 90).

37. Nancy Chodorow, The Reproduction of Mothering: Psychoanalysis and the Sociology of Gender (Berkeley, Los Angeles and London: University of California Press, 1978). 
Como fruto de su gran tesón y gallardía para asumir los retos que se le fueron presentando, en esta nueva etapa de su vida académica, fue premiada con el otorgamiento de una Monitoria de posgrado y al finalizar su maestría su tesis de grado fue evaluada con Mención Meritoria "En la maestría fui becaria, becaria de posgrado, bueno eso era una ayuda, le daban a uno un curso y le pagaban a uno el semestre. Luego fui docente ocasional y la tesis de maestría fue con Mención Meritoria".

En el historial laboral de Sonia, además, se encuentra haber laborado como docente en varias universidades ${ }^{\mathbf{3 8}}$ de reconocido prestigio a nivel nacional, entre ellas se encuentran: la Universidad Pedagógica Nacional; la Universidad Nacional de Colombia; la Universidad Antonio Nariño, la Universidad Pedagógica y Tecnológica de Colombia Sede Duitama; y la Universidad Industrial de Santander, UIS, en la ciudad de Bucaramanga, siendo esta última su actual sitio de trabajo.

Durante su carrera como docente en la UIS Sonia ha tenido la posibilidad de realizar sus estudios doctorales; participar en proyectos de alto impacto; intercambiar experiencias con especialistas nacionales y foráneos de amplio reconocimiento; publicar en revistas de gran prestigio; asistir, organizar y participar en eventos académicos nacionales e internacionales; dirigir un sinnúmero de trabajos de grado a nivel de pregrado, especialización y maestría en matemáticas y áreas afines.

Pero el camino recorrido para tener el reconocimiento y prestigio que ella hoy posee no ha sido nada fácil, ha tenido que luchar en contra de los falsos prejuicios que han afectado las dinámicas interpersonales y la valoración que tanto colegas como estudiantes han tenido al subestimar su rol como profesional de la matemática: "Recuerdo que en las dos ocasiones en que gané un concurso público docente a nivel universitario, se inventaron algunos chismes en relación a que yo había ganado gracias a relaciones sentimentales con matemáticos influyentes, cosa total y absolutamente falsa, es cierto que he tenido dichas relaciones sentimentales, pero todos mis logros académicos, pocos o muchos, grandes o pequeños, siempre los he obtenido de manera honesta, por mis propios méritos. Por otra parte, en los primeros años, sobre todo, me costaba mucho trabajo convencer a los estudiantes... o sea la actitud de ellos era como: "usted no sabe"; entonces me desgastaba un poco tratando de convencerlos de que yo sabía más o menos de lo que estaba hablando. Más con los estudiantes de los primeros semestres un poco... y con colegas también en las reuniones de escuela o las conversaciones en la cafetería... generalmente no dejan hablar a las mujeres o cuando hablan están muy pendientes de que uno cometa un error. En general los estudiantes desconfían más de las capacidades de su docente cuando es mujer que cuando se trata de un hombre".

38. Castro B. M. Sol, "La mujer docente universitaria en Colombia. Formación e inserción laboral en la segunda mitad del siglo XX", Tesis Doctoral en Doctorado Ciencias de la Educación, Universidad Pedagógica y Tecnológica de Colombia. UPTC, RUDECOLOMBIA, 2009. 


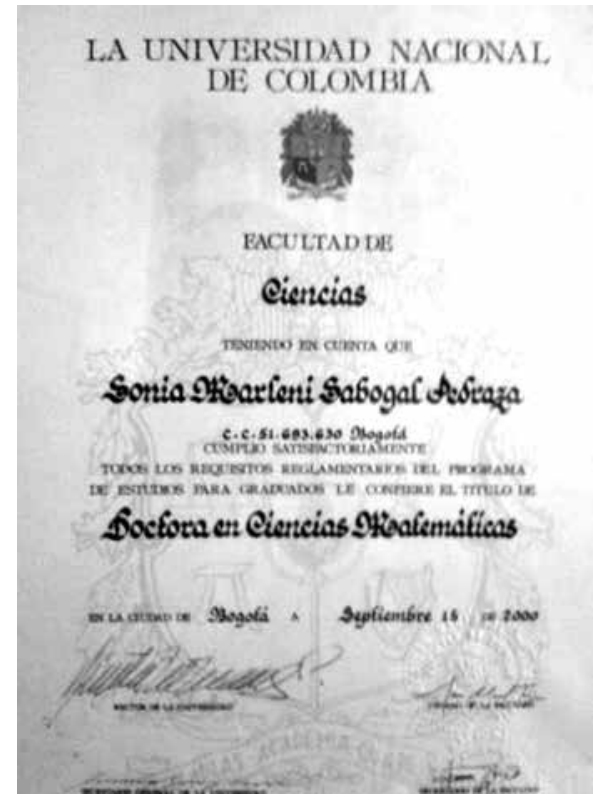

Figura 3. Diploma Doctora En Ciencias Matemáticas

Fuente: Archivo personal.
Sonia inicia su formación doctoral en la Universidad Nacional de Colombia Sede Bogotá en el año 1994, obteniendo su título de Doctora en Ciencias Matemáticas el 15 de septiembre del $2000^{39}$. De esta ardua fase de estudios ella también tiene gratos recuerdos de quienes fueran sus profesores; hace alusión al Doctor Carlos Ruíz Javier Salguero, quien además de ser su director de tesis doctoral se distinguiera por atesorar una calidez humana sin igual: "Carlos Ruiz fue mi director de tesis, él era como un papá. Él tenía un grupo grande y era muy paternal. Pero además muy amplio, o sea uno se sentaba a trabajar con él, y él le dejaba a uno hacer lo que se le ocurría, sin juzgar y luego lo orientaba, no imponía sus cosas, él lo dejaba a uno y lo motivaba a uno a que se le ocurrieran más cosas, iba escribiendo. Recuerdo que ya al final yo iba a la casa de él, estaba ya con sus años... él se sentaba en una silla y se tapaba las piernas con una cobija, uno se sentaba ahí y era como un papá. Muy diferente a sus primeros años, porque al parecer él fue un poco déspota en los primeros años".

De la misma forma, Sonia reconoce el apoyo de algunos colegas quienes coyunturalmente se convirtieron en un importante pilar que le ayudó a salir avante en sus propósitos investigativos y personales, entre ellos alude los aportes que le brindara el profesor Rafael Isaacs, quien también se desempeñaba como docente de Matemáticas en la UIS, y quien en el momento oportuno le diera un par de ideas claves en su trabajo de tesis doctoral “...Rafael también, porque él es muy apasionado también con la matemática, entonces es como un ejemplo que uno ve y que tiene cerca".

Sin embargo, independientemente de los escenarios favorables con que contó al disfrutar de la comisión de estudios que le diera la UIS para realizar su docto-

39. Sonia, junto con Clara Marina Neira Uribe, son las segundas mujeres en obtener el título de doctorado en matemáticas en la Universidad Nacional-UN (después de Margarita Ospina Pulido, quien se graduó el 14 de abril de ese mismo año). Al revisar la base de datos de doctores(as) egresados(as) de la Universidad Nacional de Colombia en Bogotá, se encuentra que Sonia posee el sexto diploma de Doctor(a) otorgado por la UN. Quienes le antecedieron fueron: José Francisco Caicedo Contreras (graduado el 28 de febrero de 1997); Néstor Raúl Pachón Rubiano (el 15 de octubre de 1999); Luis Oriol Mora Valbuena (el 14 de abril de 2000); Margarita Ospina Pulido (el 14 de abril de 2000) y Clara Marina Neira Uribe (15 de septiembre de 2000). 
rado, ella tuvo que afrontar nuevas dificultades que se le fueron presentando en el plano personal. Por aquella época sus dos hijos aún eran muy pequeños y demandaban bastante cuidado y tiempo. Para sobrellevar este conflicto, el apoyo que encontró en su familia, especialmente en una de sus hermanas, fue vital: "Pues yo tenía la comisión de la UIS, eso me ayudó mucho, porque no tenía que trabajar. Yo tuve también la suerte de que como hice el doctorado en la Nacional y estaba en comisión, en el segundo semestre ya nos fuimos para Bogotá y entonces una hermana me ayudaba, y bueno tenía una señora también que hacía el oficio, pero yo a ella no le dejaba los niños, cuando tenía que salir o ellos estaban en el colegio o si yo tenía que hacer algo, era mi hermana quien se encargaba de ellos, yo los dejaba donde mi hermana o mi hermana iba a cuidarlos en casa. El apoyo familiar que tuve en esa época y multiplicar el tiempo fue lo que me ayudó a sobrellevar estas situaciones. También durante la realización del doctorado obtuve dos becas: bueno una en realidad era una beca-crédito, otorgada por Colciencias, y la otra si era una beca de verdad, otorgada por la Fundación Mazda" ${ }^{40}$.

Tal y como Sonia lo asegura, la carrera científica demanda mucho tiempo y dedicación; si a ello se le agrega que en la idiosincrasia latina aún continúan presentes elementos machistas que obligan a la mujer a priorizar el plano familiar en detrimento de sus logros profesionales se pueden vislumbrar los agravantes a los que las mujeres de ciencia se han venido enfrentando en su desarrollo personal y profesional. Como era de esperarse, Sonia tuvo que lidiar con todos estos aspectos, por ejemplo, fue madre soltera de su primer hijo, ella tuvo que asumir sola la responsabilidad del sostenimiento de su hogar y la crianza de su primogénito, aunque reconoce que contó con apoyo logístico y moral de parte de sus padres y hermanos. Para su hija contó con mayor suerte pues el padre de la chica en gran parte asumió su deber: "con mi hija menor, el papá sí me colaboraba..., pero sí había momentos en los que yo sentía que yo llevaba más carga." A lo anterior ella agrega: “...terminar el doctorado con mis dos hijos pequeños, ;eso fue difícil!”.

Además, al preguntarle ¿Consideras que el tener hijos obstaculiza el desarrollo matemático de una mujer matemática?, ¿Cómo has afrontado la dualidad: maternidad vs. ciencia? Ella responde: "En cierta forma sí, pero no debería, podría no ser así, pero en la realidad que vivimos sí. Ahora, también puede uno tomarlo

40. La Fundación Mazda para el Arte y la Ciencia fue creada en 1991, por iniciativa de José Fernando Isaza, Presidente de la Compañía Colombiana Automotriz - CCA, como parte del compromiso con la comunidad, dentro de sus objetivos se encuentra el patrocinar y estimular la formación a nivel superior en dos áreas del conocimiento científico: la matemática pura y la física teórica; y, en la expresión artística: la música. Las becas se otorgan para estudios de posgrado en Colombia o en el exterior, en Física Teórica, Matemática Pura y Música. 
por el otro lado, tomarlo como un aliciente, que es lo que yo hacía a veces ... yo digo bueno pues voy a terminar esto por mis hijos ... (risas), voy a escribir esto para dedicárselo a mi hijo o una cosa así. Pero sí claro que se dificulta por el tiempo, sobre todo el tiempo y en ese sentido sería un obstáculo.

Por otra parte, Sonia comenta que durante su trayectoria profesional ha tenido algunas interacciones difíciles con sus colegas; actuaciones en las que ha percibido circunstancias en las que la propia comunidad matemática perpetúa la creencia de que las mujeres y las matemáticas son incompatibles: “..pues no recuerdo algo en particular, pero había cosas como muy sutiles, como que se sienten... de pronto algún comentario así, cosas así como una mirada, una expresión, ... que son cosas como muy sutiles pero uno las percibe. Ahora recuerdo también siendo profesora de una estudiante, que después nos volvimos amigas por diferentes razones, me contó y eso también me lo confirmó un colega, que ella una vez hizo una demostración que le quedó bien y fue la única del curso que la hizo, y ella la hizo sola, y se la mostró al profesor y el profesor no le creyó, le dijo: "usted no hizo esa demostración, usted no es capaz de hacer esa demostración, una mujer no es capaz de hacer esa demostración a usted se la hicieron"... y no se la valió." Estos comentarios e incidentes sexistas, que, si bien no fueron dirigidos a Sonia de manera directa, dejan claro cómo los prejuicios acerca del quehacer académico de la mujer se encuentran latentes en las aulas de clase de universidades colombianas. También aquí se hace alusión a otra circunstancia incómoda de la que Sonia tuvo conocimiento cuando era estudiante de la licenciatura: "Yo escuché el caso de un profesor que una vez le dijo a una estudiante que, ¿qué hacía ahí?, que debería estar en la casa cuidando a los hijos y haciendo el almuerzo. A mí personalmente nunca me dijeron nada de eso, pero sí lo escuché".

Así mismo, en la práctica científica Sonia ha advertido algunas actitudes que evidencian la existencia de posturas tendientes a vivificar la desigualdad de los sexos"1: "generalmente se forma un grupo de trabajo para estudiar algún tema, y casi no nos dejan hablar... o cuando se dan cuenta que uno está viendo un error o que uno se dio cuenta antes que ellos, entonces tratan de cambiar de tema y como de subestimarlo un poco y uno como ah bueno sí,... y empiezan a hablar de otra cosa ignorando un poco el tema... porque como que les da vergüenza, no sé por qué, pero es algo así".

Sonia considera que no existe diferencia alguna entre un investigador y una investigadora en matemáticas. Al preguntarle ¿Crees que hay cabida para las mu-

41. Mayor información sobre el tema en: Beauvoir, Simone (1975), ‘Entrevista con Simone de Beauvoir, para Rare TV, televisión francesa, en el programa Questionnaire, en 1975.' Entrevistada por presentador del programa Questionnaire. https://www.youtube. com/watch?v=VmEAB3ekkvU\&t=45s 
jeres en la matemática? No vacila en responder: "Sí, claro definitivamente sí. Hay muchos ejemplos y muy pocos contraejemplos". Para ella, la mujer colombiana ha participado activamente en la construcción y desarrollo del conocimiento matemático: "Pues las que están aquí, yo pienso que lo están haciendo bien. Yo conozco... son muy buenas matemáticas, muy honestas, y hay unas que han hecho aportes grandes, pero han tenido que salir ${ }^{42}$. Están en Estados Unidos, España, que yo conozca. Pero en cualquiera de los dos casos me parece que están haciendo un buen trabajo, un buen aporte".

Del impacto del trabajo científico en matemáticas de Sonia, se resalta que éste ha sido visibilizado en publicaciones realizadas en revistas internacionales de alto impacto, entre ellas está el Acta Mathematica Hungarica (formalmente Acta Mathematica Academiae Scientiarum Hungaricae, revista que publica principalmente temas en matemáticas puras, y que ocasionalmente considera artículos de naturaleza más aplicada, siempre y cuando tengan un contenido matemático no trivial. Esta revista es co-publicada con Springer). En la mencionada revista le fueron publicados los principales resultados obtenidos en su tesis de doctorado ${ }^{43}$, productos que ella considera sus primeros aportes al conocimiento matemático y que le darían cimiento a la consolidación de su carrera. Debido al prestigio que la revista mencionada tiene entre la comunidad matemática mundial, la investigación realizada por Sonia fue prontamente reconocida en el medio matemático: "Primero que se haya publicada en esa revista... esa revista es buena y me sorprendió que luego de eso me escribieron algunos, por ejemplo de Argentina me escribieron diciendo que estaban interesados en conocer mi trabajo y que les enviara lo que tuviera publicado o lo que estuviera por publicar y también en algunos eventos aquí en Colombia hay personas que están trabajando, digamos no exactamente con la extensión del teorema que hice pero sí con extensiones del mismo estilo".

42. Entre las matemáticas a que Sonia hace alusión, están: Laura Martignon. Matemática egresada de la UN en 1971. Dra. en Ciencias de la Universidad de Tubingen, Alemania. Profesora Titular de la Universidad de Ludwigsburg (Alemania). y, Directora del grupo "Matemáticas, didáctica y género" del Institut für Mathematik und Informatik, Pädagogische Hochschule de Ludwigsburg; Alemania; Nora Donaldson Matemática de la Universidad del Valle, graduada en 1974. PhD en Estadística Matemática de University of Maryland, USA, en 1988. Trabajó además en el King's College en Londres; y Tatiana Toro, egresada del programa de Matemáticas de la Universidad Nacional de Colombia en 1986. PhD de la Universidad Stanford (1992). Trabaja en el Departamento de Matemáticas de la Universidad de Washington. Merecedora de premios como: Beca Guggenheim en Ciencias Naturales, Estados Unidos y Canadá, entre otras.

43. Artículo Publicado en agosto de 2000: An Extension of the Stone Duality. Acta Mathematica Hungarica. Volume 88, Issue 3. pp. 205-211 DOI: 10.1023/A:1006761113450. Esta investigación establece una dualidad entre dos categorías, extendiendo la dualidad de Stone entre los espacios compactos, de Hausdorff y totalmente disconexos (espacios de Stone) y los anillos booleanos con 1. La primera categoría denotada por RHQS, tiene como objetos las representaciones de los cocientes Hausdorff de los espacios de Stone y como morfismos todas las funciones continuas compatibles. La segunda categoría, denotada por BRLR, tiene como objetos todos los anillos booleanos con 1, dotados de una relación de ligazón y como morfismos todos los homomorfismos de anillos de Boole con 1 , compatibles. Además, se estudia la conexidad desde un punto de vista algebraico, en el contexto de la propuesta dualidad de Stone generalizada. 
Además de los anteriores productos académicos ella también siente satisfacción por lo realizado en su momento durante la elaboración de su tesis de maestría, a la cual denominó: Propiedades de Separación y Extensiones Topológicas n-puntuales. "Aunque, como le decía antes, los trabajos de maestría son cosas muy sencillas... a mí me gustó lo que yo hice, porque lo hice sola, porque mi director no trabajaba en Topología, él trabajaba en Álgebra, sino que él resultó de director porque él quería dirigir algo en Topología, pero yo lo hacía todo como sola. En el Doctorado sí tuve un colega que me dio algunas ideas, pero también me gusta porque lo que resultó ahí fue una conexión entre Topología y Álgebra y es como una extensión de un teorema que resultó interesante para otras personas, otros grupos de investigación, uno en Argentina y uno en México."

Sonia es una apasionada por las matemáticas, las considera todo un desafío “... uno se pone a estudiar algo y salen miles de preguntas. Incluso en cada clase, así sean cosas pequeñas a mí casi siempre me queda..., la matemática es un desafío". Su entusiasmo por comprender el mundo matemático la ha llevado a profundizar en temas que le han generado inquietudes y que le han permitido apaciguar, en cierta forma, su curiosidad matemática. Dentro de sus actividades de investigación se destacan los proyectos: "Funciones Localmente Inyectivas" producido entre agosto de 1991 y febrero 1993 (con Rafael Isaacs); "Representación Algebraica de Continuos" realizado entre junio de 1998 y Julio 2000; y “Una Introducción a la Geometría Fractal a Nivel Universitario" ejecutado entre agosto de 2006 y junio de 2008 (con Gilberto Arenas); entre otros. Entre su producción académica se encuentra la elaboración de libros especializados tales como el titulado "Aproximación al álgebra lineal: un enfoque geométrico" realizado en 2009 conjuntamente con su colega Rafael Isaacs y otro realizado en 2011 junto con el profesor Gilberto Arenas, denominado "Una Introducción a la Geometría Fractal", en éste último se incluyen algunos resultados originales obtenidos por sus autores y por otros colegas con quienes tuvieron la oportunidad de departir en algunos eventos académicos.

Así mismo, fruto de su interés por el estudio de los distintos puentes que conectan la teoría de los sistemas dinámicos discretos con la teoría de los conjuntos fractales, junto con otros colegas de la UIS, durante el segundo semestre de 2007, realizó semanalmente sesiones del Seminario de Sistemas Dinámicos Discretos y Fractales, producto de este seminario fue el libro denominado Sistemas dinámicos discretos y fractales en el cual se hace una magistral exposición de los temas tratados en el seminario. 
Adicionalmente, Sonia tiene un amplio número de artículos publicados en revistas especializadas ${ }^{\mathbf{4}}$, algunos de los cuales se listan más adelante. Además de su productividad académica, Sonia manifiesta que a la par de las actividades propias de investigación disfruta mucho de la docencia universitaria. Ha impartido cursos de formación matemática a nivel de pregrado, especialización y maestría en matemáticas. También, ha actuado como tallerista con temáticas inherentes a su línea de trabajo en un abundante número de congresos, simposios y eventos académicos afines. Por ejemplo, a mediados del año 1995 orientó un "Taller de Fractales" dirigido a estudiantes de la Especialización en Educación Matemática de la UIS.

Sonia quien se define a sí misma como una persona tímida, introvertida y curiosa, desde su vocación como docente universitaria ha tenido la oportunidad de trascender en sus estudiantes. Particularmente se ha convertido en el ejemplo a seguir que tienen todas y cada una de las chicas que transitan por sus aulas de clase; o de aquellas otras que han presenciado sus intervenciones en eventos científicos especializados; o de quienes han leído sus publicaciones. Al respecto, ella expresa que ha tenido experiencias muy enriquecedoras con algunas de quienes en algún momento fueron sus estudiantes.

A la pregunta: ¿Recuerdas alguna estudiante quien te haya expresado abiertamente que tu desempeño profesional en Matemáticas representa un ejemplo a seguir? Sin titubear responde: "sí, me ha pasado. Y me he seguido hablando con ellas. Bueno no con todas, pero hay una que está trabajando ahorita en Barranca. Bueno, lo que pasa es que como aquí hasta ahora se abrió la maestría en matemáticas... y parece que se va abrir el doctorado, entonces algunas se han ido a Bogotá, algunas a Brasil. Pero de las que me han dicho eso o algo de ese estilo... con algunas no me volví a ver, pero hay dos o tres que sí me llaman a veces y me cuentan lo que están haciendo".

Para Sonia, en Colombia actualmente existen las condiciones para el desarrollo de las potencialidades de las mujeres en las matemáticas se den; claro que según ella:

“... podrían mejorar y deberían mejorar, pero si existen”.

Desde su óptica y experiencia vivida, Sonia sugiere a las jóvenes: “A las chicas que posiblemente estén interesadas en incursionar en el estudio de las matemáticas, o que están iniciando dicho estudio, les diría en primer lugar algo muy sencillo y casi que obvio pero que quizá a veces no se prioriza lo suficiente, y es que, en mi opinión, para escoger una carrera (y en general para escoger cualquier actividad en la vida), lo más importante es que a uno

44. Sin embargo, el impacto académico de su producción, visto desde el índice de citación de sus trabajos no es el deseado; por ejemplo, al realizar una búsqueda de citaciones en el Google Académico y estimar el índice h de Hirchs de 12 de sus productos más citados, encontramos un índice h de 2, lo cual significa que apenas dos de sus productos tienen al menos dos citas. Este índice resultaría bajo si se compara con el promedio mundial para el área de matemáticas que es de 17. 
le guste; así de sencillo; debe haber como mínimo un gusto por la matemática, un disfrute, ese gusto o disfrute se irá transformando en pasión y verdadero amor (si es que de entrada ya no lo es); esa pasión y amor implicarán mucha constancia, dedicación, disciplina, gozo, diversión y también a veces sufrimientos, temores y frustraciones, pero hay que mantener el gusto y por tanto el entusiasmo y la motivación. La matemática es un mundo fascinante, en constante evolución, lleno de sorpresas y bellezas. Habrá que hacer algunos sacrificios, sobre todo en los primeros años, estudiar mucho, organizar muy bien el tiempo para poder responder no solo con el estudio, sino también con otros compromisos o actividades inherentes a la vida, por ejemplo, de carácter familiar, personal, laboral, espiritual, social, en nuestro caso específico de mujeres, la maternidad, etc.... etc. Por supuesto y naturalmente se presentarán obstáculos, problemas, dificultades de diversa índole, y esto, infortunadamente aún hoy en día ocurre más para las mujeres que para los hombres (espero que esta situación definitivamente cambie algún día), pero mi consejo es que hay que persistir, tomar aliento, ser solidarias, optimistas y tomar esos obstáculos como valiosas oportunidades de aprender, de conocerse a una misma y fortalecerse más. Con el tiempo las cosas se van solucionando, alegrías y tristezas, logros y fracasos van fluyendo bien, armoniosamente... como deben fluir las cosas en una buena historia de amor... para mi esas son las buenas historias de amor".

Finalmente, se anexa, una pequeña muestra de la productividad científica de nuestra matemática:

- "Filtros y Extensiones Topológicas". En: Lecturas Matemáticas Ed: Sociedad Colombiana de Matemáticas, 1989 (con Víctor S. Albis. Q.E.D).

- "Separation Properties and n-point Topological Extensions". En: Revista Colombiana De Matemáticas Ed: Sociedad Colombiana de Matemáticas, 1990 (con Víctor S. Albis, Q.E.D).

- "Dos observaciones elementales sobre la Ti-clausura de espacios topológicos". En: Lecturas Matemáticas Ed: Sociedad Colombiana de Matemáticas, 1990.

- "Pliegues en la recta real". En: Lecturas Matemáticas Ed: Sociedad Colombiana de Matemáticas, 1992.

- "Semigrupo de funciones localmente inyectivas sobre $\mathrm{S}^{1}$ ". En: Lecturas Matemáticas Ed: Sociedad Colombiana de Matemáticas, 1994 (con Rafael F. Isaacs).

- "Funciones localmente inyectivas y pegamientos". En: Lecturas Matemáticas Ed: Sociedad Colombiana de Matemáticas, 1994.

- "Acerca del recíproco del lema de Schur". En: Boletín de Matemáticas Ed: Sociedad Colombiana de Matemáticas, 1996 (con Víctor Ardila). 
- “Sobre Auto semejanza Topológica. Parte I." En: Revista Integración. Ed: Ediciones Universidad Industrial De Santander, 1999.

- "An Extension of the Stone duality". En: Acta Mathematica Hungarica. Hungría, 2000.

- "An extension of the Stone duality: the expanded version". En: Boletín de Matemáticas. Ed: Sociedad Colombiana de Matemáticas, 2006.

- "Algebraic Representation of continua". En: Revista Colombiana de Matemáticas Ed: Sociedad Colombiana de Matemáticas, 2007.

- "Acerca del Triángulo de Sierpinski". En: Revista de la Academia Colombiana de Ciencias Exactas, Físicas y Naturales. Ed: ACCEFYN, 2009.

\section{Conclusiones}

En esta investigación, desde la postura de género ${ }^{45}$ en la historia social de la educación, y con la metodología inherente a la historia oral, podemos destacar en primera instancia que la historia de vida de la participante en este estudio, muestra que las mujeres si han estado presentes en la historia de las matemáticas en Colombia, han realizado aportes tanto en la producción de conocimiento nuevo en matemáticas, como en la labor de formadoras de nuevos(as) matemáticos(as) colombianos(as). Un segundo factor observable es que no han tenido ni el reconocimiento, ni la visibilización, que merecen porque han estado tan inmersas en un mundo idealizado por la perspectiva masculina, que en algunas ocasiones pasa desapercibida por algunas mujeres. Desde esta óptica, esta investigación procuró tratar de entender las experiencias de las mujeres en matemáticas y cómo esas experiencias están ligadas a la cultura y las creencias de la comunidad matemática.

Las experiencias contadas por nuestra investigadora participante nos develan que en la comunidad matemática colombiana persisten los mitos e imaginarios que invisibilizan la labor científica de las mujeres matemáticas. Además, el trabajo académico llevado a cabo por mujeres como Sonia, evidencia el papel que desempeña la mujer en el contexto universitario, en escenarios cuyo ingreso tubo variados tropiezos ${ }^{46}$.

Los procesos investigativos desarrollados por nuestra participante muestran que sí existe presencia de las mujeres matemáticas colombianas en la producción y reproducción de ésta ciencia, y, además, permite rescatar de la oscuridad a in-

45. Joan W. Scott, Género e historia (México D.F.: Editorial Fondo de Cultura Económica, 2008).

46. Ana Hercilia Hamón Naranjo, "Las mujeres docentes en la Universidad Pedagógica y Tecnológica de Colombia 1961-1980", Tesis Doctoral, Universidad Pedagógica y Tecnológica de Colombia, Doctorado en Ciencias de la Educación. RUDECOLOMBIA. Tunja, 2010. 
vestigadoras que han hecho contribuciones significativas de forma asidua, pero que no han logrado la reputación y el reconocimiento que merecen.

Los logros matemáticos de Sonia, y el hecho de ser una de las dos primeras licenciadas, junto con Clara Marina Neira Uribe, en doctorarse en matemáticas en Colombia, muestran que la formación como licenciadas no es una limitante para avanzar en una ciencia netamente teórica y abstracta, rodeada de imaginarios ${ }^{47}$, como lo es la matemática. Ambas, Sonia y Clara Marina, son egresadas del Programa de Licenciatura con estudios principales en Matemáticas, de la Universidad Pedagógica Nacional, en Bogotá. Sin lugar a dudas, ellas han marcado una impronta inspiradora para otras mujeres.

Al analizar la historia de vida de la participante de esta investigación, diferimos de la problemática planteada por Herion (1997) ${ }^{48}$ al afirmar que las mujeres son más propensas que los hombres a sentirse marginadas; y que es más probable que tengan menores niveles de confianza en sí mismas, y que tengan más probabilidades de encontrar obstáculos sutiles u obvios para su desarrollo, aceptación y reconocimiento. En el caso de nuestra entrevistada, no se evidenció este problema, por el contrario, ella se caracterizó por poseer un talante excepcional, característico de la mayoría de mujeres colombianas. Las mujeres colombianas son admirables, tanto que son cabeza de la tercera parte de los hogares de nuestro país ${ }^{49}$.

A pesar del importante avance de la mujer en las últimas décadas, aún está lejos de ocupar el espacio que le corresponde en las ciencias en general, y en las matemáticas en particular; prueba de ello es que apenas el pasado 13 de agosto de 2014, por primera vez en la historia, una mujer es premiada con la medalla Fields de Matemáticas ${ }^{50}$, esta medalla es considera como "el premio Nobel de Matemáticas". La Medalla Fields fue instaurada en 1936 y cada cuatro años durante la celebración del Congreso Internacional de Matemáticas premia, por sus descubrimientos sobresalientes, a un máximo de cuatro matemáticos menores de 40 años. En esta ocasión la medalla fue otorgada a la matemática iraní Maryam Mirzakhani, Q.E.D.51, de 37 años y como se mencionó anteriormente, por primera vez en la historia de las matemáticas este premio es otorgado a una mujer. La actual presiden-

47. L. Figueroa, M. Molero, A. Salvador y N. Zuasti, Género y Matemáticas (Madrid: Síntesis, 1998).

48. Claudia Herion, Women in Mathematics: The Addition of Difference Race, Gender, and Science (U.S.A.: Indiana University Press, 1997).

49. The New York Times (1994), 'Women in Colombia Move to Job Forefront', http://www.nytimes. com/1994/07/15/world/women-in-colombia-move-to-job-forefront.html?pagewanted=all. Redactado por James Brooke. Publicado el 15 de junio de 1994. versión Online (13 de mayo de 2016)

50. La información completa de este suceso se puede leer en: http://sociedad.elpais.com/ sociedad/2014/08/13/actualidad/1407915530_921490.html (13 de agosto de 2014).

51. Paradójicamente, la profesora de la Universidad de Stanford, Maryam Mirzakhani falleció el 14 de julio de 2017 a raíz de un cáncer de mama que padecía desde hacía cuatro años atrás. Información ampliada en http://www.elmundo.es/ciencia-y-salud/ciencia/2017/07/15/5969f89822601d5c378 b469a.html 
ta de la Unión Matemática Internacional, IMU, Ingrid Daubechies, ha reconocido que "las mujeres siguen sin estar lo suficientemente presentes en la investigación matemática, por lo que Mirzakhani es un modelo para atraer a más féminas a los primeros puestos"52. El Congreso Internacional de Matemáticas de 2014 pasará a la historia por la coincidencia de dos efemérides relacionadas con las mujeres: la primera mujer presidente de la IMU presidió la ceremonia de entrega de la Medalla Fields a la primera mujer matemática receptora del tan anhelado galardón, considerado el Premio Nobel de Matemáticas y el premio más prestigioso en el campo de las matemáticas.

52. Award Ceremony for the Fields Medals during the International Congress of Mathematicians 2014 in Seoul. https://www.youtube.com/watch?v=TxbE6mkYUAg (13 de agosto de 2014). 


\section{REFERENCIAS BIBLIOGRÁFICAS}

\section{Fuentes primarias}

Beauvoir, Simone, "Entrevista con Simone de Beauvoir, para Rare TV, televisión francesa, en el programa Questionnaire, en 1975". Entrevistada por el presentador del programa Questionnaire. Recuperada de https://www.youtube. com/watch?v=VmEAB3ekkvU\&t=45s.

Mantilla Prada, Eduardo, “Entrevista Historia del Departamento de Matemáticas de la Universidad Nacional de Colombia". Entrevistada por Nelsy Rocío González Gutiérrez. En persona. Realizada el 22 de enero de 2016 en Bogotá D.C., Colombia.

Sabogal Pedraza, Sonia Marleni, "Entrevista Historia de Vida”, Entrevistada por Nelsy Rocío González Gutiérrez. En persona. Realizada el 8 de mayo de 2016 en Bucaramanga, Colombia.

\section{Archivos, memorias y documentos oficiales}

Barrantes, Hugo \& Ruiz, Ángel, 'La historia del Comité Interamericano de Educación Matemática', Academia Colombiana de Ciencias Exactas físicas y Naturales. Colección Enrique Pérez Arbeláez. No. 13. Santafé de Bogotá, 1998. Disponible en la web: http://www.centroedumatematica.com/aruiz/libros/ La\%20Historia\%20del\%20Comite\%20Interamericano\%20de\%20Educacion\% 20Matematica.pdf

Conversatorio: "Mujeres matemáticas en Colombia. Algunos testimonios". Dentro del XXI Congreso Colombiano de Matemáticas realizado en la sede Bogotá de la Universidad Nacional de Colombia entre el 5 y el 9 de junio de 2017. Disponible en https://scm.org.co/scm2017/2017/07/18/conversatorio-mujeresmatematicas-en-colombia-algunos-testimonioso-lineal-y-ecuaciones-diferenciales-parciales-2017-2/

Diario El País, “Por primera vez en la historia, una mujer gana la medalla Fields de Matemáticas. La iraní Maryam Mirzakhani, de 37 años, recibe el que se considera el Nobel de estas ciencias. Entre los otros tres galardonados, un brasileño: primer latinoamericano en obtener la distinción". https://elpais.com/sociedad/2014/08/13/actualidad/1407915530_921490.html Redactado el 13 de agosto de 2014. THE SEOUL ICM 2014 (AFP).

Garzón Barreto, Juan Carlos, “El proceso de consolidación de la Universidad Pedagógica Nacional Femenina en el marco de la contrarreforma educativa de los años 50", Publicaciones de la Universidad Pedagógica Nacional (s.f). http://www.pedagogica.edu.co/storage/rce/articulos/rce36-37_05ensa.pdf

Organización de Naciones Unidas, Día Internacional de la Mujer y la Niña en la Ciencia, 11 de febrero. Disponible en http://www.un.org/es/events/women-and-girls-in-science-day/

Presidencia de la República de Colombia, "Decreto No 1930 del 06 de septiembre de 2013, por el cual se adopta la política pública nacional de equidad de género y se crea una comisión intersectorial para su implementación", Departamento Administrativo para la Prosperidad Social, 2013. Disponible en la web: http://www.equidadmujer.gov.co/ejes/Documents/decreto-1930.pdf

SCM, Foro: Las Matemáticas en Colombia, Universidad Nacional de Colombia sede Manizales y Sociedad Colombiana de Matemáticas, 2015. SCM, XX Congreso Colombiano de Matemáticas, julio 21-24. https:// www.youtube.com/ watch?v=QQh3HG9tNs4\&feature=youtu.be

Secretaría de Naciones Unidas, “Informe de la cuarta conferencia mundial sobre la mujer", 1995. http:// www.un.org/womenwatch/daw/beijing/pdf/Beijing\% 20full\%20report\%20S.pdf

Secretaría Facultad de Ciencias - Universidad Nacional de Colombia, "Base de datos de graduados(as) de la Carrera de Matemáticas, y de los posgrados de Maestría y Doctorado en Matemáticas", Secretaría Facultad de Ciencias, 2016. Contiene los datos de egresados desde 1961 hasta 2016.

UNESCO, “Organización de las Naciones Unidas para la Educación la Ciencia y la Cultura”, 1998. http:// www.unesco.org/new/es/natural-sciences/ priority-areas/gender-and-science/for-women-in-scienceprogramme/ 
Universidad Nacional de Colombia, "Proyecto Educativo del Programa de Matemáticas”, 2014. http://www. pregrado.unal.edu.co/docs/pep/pep_2_3.pdf

\section{Prensa}

Padrón, Edith \& Timón, Ágata A., “¿Dónde están las mujeres matemáticas?”, Día Internacional de la Mujer. Sección Café y Teoremas, El País, Edición América, jueves 9 de marzo de 2017. http://elpais.com/elpais/ ciencia 2017/03/07/

Periódico El Tiempo, “Doctorado de Matemáticas. Con cuatro líneas de investigación, la Universidad Nacional de Colombia ofrece en Bogotá desde 1993 el primer doctorado de matemáticas en el país”. Disponible en http://www.eltiempo. com/archivo/documento/MAM-553220. Publicado el 9 de marzo de 1997, versión Online, 13 de febrero de 2017.

Periódico El Tiempo, "Entrevista con Peter Gruss, presidente de la Sociedad Max Planck”, Disponible en http://www.eltiempo.com/archivo/documento/ CMS-13156067. Redactado por Andrea Linares Gómez, publicado el 2 de noviembre de 2013, versión Online; último acceso 20 de mayo 2016.

Periódico El Tiempo, “Mujeres”, Disponible en http://www.eltiempo.com/ archivo/documento/MAM1270739. Redactado por Gonzalo Gallo G. el 20 de julio de 2000, versión Online, último acceso 19 mayo 2016.

Periódico El Tiempo, “Los intensos años sesenta. Capítulo del libro de Álvaro Tirado Mejía”, 2014, versión digital disponible en http://www.eltiempo.com/lecturas-dominicales. Extraído del libro: Los años 60, una revolución en la cultura.

Revista CIMPEC. Publicación para el cuerpo docente de América Latina, 'La mujer y la matemática', Centro Interamericano para la Producción de Material Educativo y Científico para la Prensa. Año 12, Número 47. Abril - Junio, 1985.

The New York Times, “Women in Colombia Move to Job Forefront”, http://www.nytimes.com/1994/07/15/ world/women-in-colombia-move-to-job-forefront.html?pagewanted=all. Redactado por James Brooke. Publicado el 15 de junio de 1994.

\section{Referencias}

Alic, M. El Legado de Hipatia. Historia de las mujeres en la ciencia desde la antigüedad hasta fines del siglo XIX, primera edición, México DF: Siglo XXI editores, s.a. de c.v., 2005.

Appel, M., "La entrevista autobiográfica narrativa: Fundamentos teóricos y la praxis del análisis mostrada a partir del estudio de caso sobre el cambio cultural de los Otomíes en México [127 párrafos]", Forum Qualitative Sozialforschung / Forum: Qualitative Social Research 6(2), (2005): 1-35. http://nbn-resolving.de/ urn:nbn:de:0114-fqs0502160.

Castro B., Sol M. "La mujer docente universitaria en Colombia. Formación e inserción laboral en la segunda mitad del siglo XX”, Tesis doctoral, Doctorado Ciencias de la Educación, Universidad Pedagógica y Tecnológica de Colombia. UPTC, RUDECOLOMBIA, 2009.

Chodorow, Nancy. The Reproduction of Mothering: Psychoanalysis and the Sociology of Gender. Berkeley, Los Angeles and London: University of California Press, 1978.

Clair, Rene. La Formación científica de las mujeres. ¿Por qué hay tan pocas científicas? Barcelona: Ediciones UNESCO, 1994.

Cooke, Roger. The History of Mathematics, A Brief Course. New Jersey: John Wiley y Sons, Inc., 2005.

Creswell, J. W. Qualitative inquiry research desing. Choosing among five approaches, first edn. United States of America: SAGE Publications Inc., 2013.

De Sousa Santos, B., Meneses, M. \& Bonet, A. Epistemologías del Sur, cuestiones de antagonismo, Madrid: Ediciones Akal, 2014, https://books.google. com.co/books?id=rfEmnwEACAAJ

Deslauriers, J. P. Investigación Cualitativa. Guía Práctica, Universidad Tecnológica de Pereira, Rudecolombia, Traducción de Miguel Ángel Gómez, 2004. 
Figueroa, L., Molero, M., Salvador, A. y Zuasti, N. Género y Matemáticas, Madrid: Síntesis, 1998.

Frías Ruiz, V., ed., Las mujeres ante la ciencia del siglo XXI, Madrid: Instituto de Investigaciones Feministas, Editorial Complutense S.A., 2001.

González G. Nelsy R. “Investigadoras en Ciencias Matemáticas en la universidad colombiana. 1961-2000”, Tesis Doctoral Doctorado en Ciencias de La Educación. RUDECOLOMBIA, Universidad Pedagógica y Tecnológica de Colombia, 2017.

Guha, Ranahit. Las voces de la historia y otros estudios subalternos. Barcelona: Editorial Crítica, 2002.

Hamón Naranjo, A. H. Las mujeres docentes en la Universidad Pedagógica y Tecnológica de Colombia 19611980, Tesis Doctoral Doctorado en Ciencias de la Educación. RUDECOLOMBIA, Universidad Pedagógica y Tecnológica de Colombia, 2010.

Harding, S. The Science Question in Feminism, United States of America: Cornell University Press, 1986.

Herion, Claudia. Women in Mathematics: The Addition of Difference Race, Gender, and Science. U.S.A: Indiana University Press, 1997.

Inter-American Network of Academies of Sciences - IANAS, "Jóvenes científicas: Un futuro brillante para las Américas", Traducido por Suzanne D. Stephens, ISBN: 978-607-8379-24-8. Impreso en México, 2015. http://www.ianas.org/women_2015/young_women_scientists/libro-jovenes-cientificas.pdf

Lafuente Guantes, M. I., "El proyecto educativo-ilustrado de Kant" Revista Historia de la Educación Latinoamericana 13 (2009): 241-264. http://www.redalyc.org/articulo.oa?id=86912384013.

SCM, Foro: Las Matemáticas en Colombia, Universidad Nacional de Colombia sede Manizales y Sociedad Colombiana de Matemáticas. SCM, XX Congreso Colombiano de Matemáticas, julio 21-24, 2015. https:// www.youtube.com/watch?v=QQh3HG9tNs4\&feature=youtu.be

Scott, Joan W. , "Gender: A useful category of historical analysis", The American Historical Review 91(5) (1986): 1053-1075. Versión digital disponible en https://racismandnationalconsciousnessresources.

Scott, Joan W., Género e Historia, México D.F: Editorial Fondo de Cultura Económica, 2008.

Soto Arango, Diana Elvira, "La escuela rural en Colombia: Historias de vida de maestras. Mediados del siglo XX", Historia de la Universidad Latinoamericana. Tunja: grupos HISULA, ILAC, SHELA (2014): 51-58.

Soto Arango, Diana Elvira, "Aproximación histórica a la universidad colombiana", Revista Historia de la Educación Latinoamericana 7 (2005): 99-136. Tunja: Universidad Pedagógica y Tecnológica de Colombia.

Vasilachis et al. Estrategias de Investigación cualitativa, Barcelona: Gedisa, S.A., 2006. 